\title{
Transfection Studies with Colloidal Systems Containing Highly Purified Bipolar Tetraether Lipids from Sulfolobus acidocaldarius
}

\author{
Konrad H. Engelhardt, Shashank Reddy Pinnapireddy, Elias Baghdan, \\ Jarmila Jedelská, and Udo Bakowsky
}

Department of Pharmaceutics and Biopharmaceutics, University of Marburg, Marburg, Germany

Correspondence should be addressed to Udo Bakowsky; ubakowsky@aol.com

Received 1 September 2016; Revised 4 December 2016; Accepted 22 December 2016; Published 23 January 2017

Academic Editor: Nejat Düzgünes

Copyright (C) 2017 Konrad H. Engelhardt et al. This is an open access article distributed under the Creative Commons Attribution License, which permits unrestricted use, distribution, and reproduction in any medium, provided the original work is properly cited.

\begin{abstract}
Lipid vectors are commonly used to facilitate the transfer of nucleic acids into mammalian cells. In this study, two fractions of tetraether lipids from the archaea Sulfolobus acidocaldarius were extracted and purified using different methods. The purified lipid fractions polar lipid fraction E (PLFE) and hydrolysed glycerol-dialkyl-nonitol tetraether (hGDNT) differ in their structures, charge, size, and miscibility from conventional lipids. Liposomes were prepared by mixing tetraether lipids with cholesterol $(\mathrm{CH})$ and 1,2-dioleoyl-3-trimethylammonium-propane (DOTAP) resulting in stable vectors for gene delivery. Lipoplexes were prepared by complexation of liposomes with a luciferase expressing plasmid (pCMV-luc) at certain nitrogen-to-phosphorus (N/P) ratios and optimised for the transient transfection of ovarian adenocarcinoma cells (SK-OV-3). Complexation efficacy was investigated by gel-red fluorescence assay. Biophysical properties, like size, surface charge, and morphology, were investigated by differential light scattering (DLS), atomic force microscopy (AFM), and scanning electron microscopy (Cryo-SEM), respectively, revealing structural differences between liposomes and lipoplexes. A range of stable transfecting agents containing tetraether lipids were obtained by incorporating $5 \mathrm{~mol} \%$ of tetraether lipids. Lipoplexes showed a decrease in free gel-red with increasing N/P ratios indicating efficient incorporation of plasmid DNA (pDNA) and remarkable stability. Transfection experiments of the lipoplexes revealed successful and superior transfection of SK-OV-3 cell line compared to the commercially available DOTAP and branched polyethyleneimine (25 kDa bPEI).
\end{abstract}

\section{Introduction}

At present, transfection vectors can be broadly classified into viral and nonviral. Since early days of gene therapy, nonpathogenic attenuated viruses have been the most common delivery systems used. Currently, viral vectors are employed in over $65 \%$ of gene therapy clinical trials worldwide, with adenoviruses being the most common, followed by retroviruses. Viruses possess an innate ability to insert their own genetic material into the host cell; therefore, high transfection efficiency is usually observed in a variety of human tissues. Despite this significant advantage, there are several concerns over the safety of viral vectors. These include the potential to induce a potent, possibly lethal immune response, generation of replication competent virus, and producing insertional mutagenesis due to random gene transfer that can lead to oncogenesis. Additionally, the capacity of viral vectors is restricted, which limits the size of genetic material that the vectors can accommodate. Nonviral vectors like liposomes offer promising alternatives for the delivery of DNA-based biopharmaceuticals [1-3] However, several problems still need to be addressed before liposomes can be used in a clinical setting. One of the major problems is poor stability and integrity of liposomes, which is partially attributed to the hydrolysis of ester bonds and oxidation of unsaturated fatty acids [4]. Tetraether lipids (TELs) are a unique class 
of lipids found exclusively in the cellular membranes of members of the third domain of life referred to as archaea, which can be considered promising alternatives to common lipids for liposomes intended for drug delivery. Members of the archaea are known to inhabit environments ranging from ordinary to those characterised by extremes of salt, temperature, and $\mathrm{pH}$, for example, hypersaline and alkaline lakes, hot acid springs, and strictly anoxic settings [5-7]. The presence of ether lipids in membranes of the archaea is believed to be responsible for their unusual temperature and $\mathrm{pH}$ stability. Ether lipids differ considerably from prokaryotic and eukaryotic lipids, the main difference being the presence of hydrocarbon moieties linked to a glycerol/nonitol bridge group by an ether bond, which give the lipid molecule more resistance against hydrolytic damage than ester bonds in extremes of $\mathrm{pH}$ and temperature. Another difference is the structure of the hydrocarbons themselves; archaeal lipids consist of branched saturated isoprenoids, the most common being the $\mathrm{C}_{20}$ phytanyl, as opposed to straight chain unsaturated hydrocarbons in prokaryotic and eukaryotic membrane lipids. The lack of unsaturation protects ether lipids from oxidative damage [8]. Ether lipids can be broadly classified into two groups: monopolar and bipolar. Monopolar ether lipids (diether lipids) somewhat resemble common lipids in their structure, having two hydrocarbon chains linked by a bridge moiety to a single head group. Bipolar TELs are unique due to the presence of two head groups, one at each end of the molecule. They resemble two diether lipids whose tails have been covalently bound and are present in a monolayer in archaeal membranes. The monolayer arrangement is believed to add to the thermal and $\mathrm{pH}$ stability of the lipid membrane. TELs can be further subdivided into two subclasses, namely, glycerol-dialkyl-glycerol tetraether (GDGT) or glycerol-dialkyl-nonitol tetraether (GDNT) lipids. GDNTbased tetraethers constitute $70-80 \%$ or more of the total lipids of thermoacidophilic archaea such as Sulfolobus acidocaldarius $[9,10]$. One of the promising applications of TEL liposomes is oral drug delivery owing to their stability in extreme $\mathrm{pH}$ conditions [10], as well as in the presence of serum, lipases, and bile salts. TEL liposomes have been proven to be a good carrier for the oral delivery of peptides like insulin [11], skin applications [12], and chlorine e6 for photodynamic therapy [13]. Tetraether lipids can be modified to obtain site specific interactions [14]. For gene delivery, synthetic or semisynthetic tetraether as well as diether lipids has been used [15-17]. In this study, native purified nonhydrolysed tetraether lipids (PLFE) and their hydrolysed backbones (hGDNT) from the archaeon Sulfolobus acidocaldarius were combined with the helper lipids cholesterol $(\mathrm{CH}), \mathrm{L}-\alpha$-phosphatidylcholine (PC), and a cationic lipid 1,2dioleoyl-3-trimethylammonium-propane (DOTAP) to prepare stable liposomes. Lipoplexes were then prepared by complexation with a luciferase expressing plasmid (pCMVluc). The effectiveness of lipoplexes as gene delivery vehicles was investigated by performing in vitro transfection studies in SK-OV-3 cell line and compared against branched polyethyleneimine ( $25 \mathrm{kDa}$ bPEI). Furthermore, the delivery vehicles were rated in relation to their ability to be used in oral gene delivery.

\section{Materials and Methods}

2.1. General Materials. Freeze-dried biomass of Sulfolobus acidocaldarius was obtained from SiT (Surface \& Interface Technologies, Rosenhof GmbH, Heiligenstadt, Germany). N-[1-(2,3-Dioleoyloxy)propyl]-N,N,N-trimethylammonium chloride (DOTAP) was a gift from Lipoid (Ludwigshafen, Germany). L- $\alpha$-Phosphatidylcholine was purchased from Avanti Polar Lipids (Alabama, USA) and cholesterol was obtained from Sigma-Aldrich (Taufkirchen, Germany). DNA intercalating dyes gel-red (10.000x in DMSO) and ethidium bromide (EtBr) were obtained from Invitrogen (California, USA) and Sigma-Aldrich (Missouri, USA), respectively. Purified plasmid pCMV-luc (pDNA) was purchased from Plasmid Factory (Bielefeld, Germany). Silica gel 60 (400-230 mesh) was obtained from Carl Roth GmbH (Karlsruhe, Germany). Reversed phase Chromabond C-18 and HPTLC (high performance thin layer chromatography) plates were bought from Macherey-Nagel (Weilmünster, Germany). Organic solvents chloroform $\left(\mathrm{CHCl}_{3}\right)$, methanol $(\mathrm{MeOH})$, and diethyl ether (DE) were obtained from VWR International (Pennsylvania, USA). Hydrochloric acid was purchased from Sigma-Aldrich. For $1 \mathrm{H}-\mathrm{NMR}$, deuterated chloroform $\left(\mathrm{CDCl}_{3}\right)$ was obtained from Euriso-Top (Gif-surYvette Cedex, France). All solvents used were of HPLC grade.

2.2. Cell Culture. SK-OV-3 adenocarcinoma ovarian cells were purchased from ATCC (Virginia, USA) and cultured in Iscove's Modified Dulbecco's Medium (IMDM), supplemented with $10 \%$ fetal calf serum. Cells were maintained as monolayers at $37^{\circ} \mathrm{C}$ with $5 \% \mathrm{CO}_{2}$ and subcultured upon reaching $80 \%$ confluency. For transfection studies, Luciferase Cell Culture Lysis 5x Reagent and luciferin were purchased from Promega (Wisconsin, USA). Pierce BCA Assay was purchased from Thermo Scientific (Massachusetts, USA).

\subsection{Isolation and Purification of Tetraether Lipids}

2.3.1. Polar Lipid Fraction E (PLFE). $6.3 \mathrm{~g}$ of freeze-dried biomass of Sulfolobus acidocaldarius was pestled for $15 \mathrm{~min}$ until a coarse fine yellow/greenish powder was obtained. It was then transferred into an extraction thimble for Soxhlet extraction. $500 \mathrm{~mL}$ mixture of $\mathrm{CHCl}_{3}: \mathrm{MeOH}(50: 50 \mathrm{v} / \mathrm{v})$ was poured into a $1000 \mathrm{~mL}$ round bottom flask (RBF). Extraction was performed for $48 \mathrm{~h}$ using an oil bath at $80^{\circ} \mathrm{C}$. After extraction, the organic solvent was evaporated leaving a crude lipid layer on the flask's wall. The crude lipid extract was then suspended in a small volume of $\mathrm{MeOH}: \mathrm{H}_{2} \mathrm{O}$ $(50: 50 \mathrm{v} / \mathrm{v})$ using an ultrasonic bath, Elmasonic P30 H from Elma Hans Schmidbauer, (Singen, Germany). The suspension was then transferred to a Chromabond C-18 column. The eluents used for the purification process were $\mathrm{MeOH}: \mathrm{H}_{2} \mathrm{O}$ $(50: 50 \mathrm{v} / \mathrm{v}), \mathrm{CHCl}_{3}: \mathrm{MeOH}: \mathrm{H}_{2} \mathrm{O}(22.5: 55: 22.5 \mathrm{v} / \mathrm{v})$, and $\mathrm{CHCl}_{3}: \mathrm{MeOH}: \mathrm{H}_{2} \mathrm{O}(70: 26: 4 \mathrm{v} / \mathrm{v})$.

2.3.2. Hydrolysed Lipid (hGDNT). $0.5 \mathrm{~g}$ of freeze-dried biomass of Sulfolobus acidocaldarius was pestled and suspended in $4 \mathrm{M} \mathrm{HCl}$ in $500 \mathrm{~mL}$ RBF. The flask was put onto a reflux condenser for $24 \mathrm{~h}$ at $100^{\circ} \mathrm{C}$. The suspension was filtered 
through a G-4 frit and the resulting filter cake was dried before being extracted five times with $50 \mathrm{~mL}$ chloroform at room temperature. The extract was concentrated and transferred onto a silica-gel column. Lipid material was purified by using the solvents $\mathrm{CHCl}_{3}, \mathrm{CHCl}_{3}: \mathrm{DE}(80: 20 \mathrm{v} / \mathrm{v})$, and $\mathrm{CHCl}_{3}: \mathrm{MeOH}(90: 10 \mathrm{v} / \mathrm{v})$. The last eluent was crucial in obtaining purified hGDNT.

2.4. Analysis of Tetraether Lipids. The purified hydrolysed lipids were analysed by mass spectrometry using a Q-Trap 2000 (Applied Biosystems, Foster City, USA) which operated at ion-spray ionisation (ESI-MS). The purified lipid hGDNT was diluted to $0.1 \mu \mathrm{g} / \mathrm{mL}$ prior to measurement. IR spectrum of hGDNT was recorded by an ALPHA FT-IR spectrometer (Bruker Corp., Massachusetts, USA). $5 \mathrm{mg}$ of hGDNT was used to obtain the transmission spectrum. For $1 \mathrm{H}-\mathrm{NMR}$ studies, hydrolysed lipids were dissolved at $2.5 \mathrm{mg} / \mathrm{mL}$ in $\mathrm{CDCl}_{3}$ and analysed with a JEOL ECX-400 with an autotune sample head. HPTLC plates were used to identify the lipids PLFE and hGDNT using the mobile phases $\mathrm{CHCl}_{3}: \mathrm{MeOH}: \mathrm{H}_{2} \mathrm{O}$ $(22.5: 50: 22.5 \mathrm{v} / \mathrm{v})$ and $\mathrm{CHCl}_{3}: \mathrm{MeOH}(90: 10 \mathrm{v} / \mathrm{v})$, respectively. Lipids were spotted using a $\mathrm{MeOH} /$ sulfuric acid spray reagent.

2.5. Preparation of Liposomes. Liposomes were prepared using the film hydration method [18]. Stock solutions of lipids were prepared by dissolving the lipids in a $\mathrm{CHCl}_{3}: \mathrm{MeOH}$ $(2: 1 \mathrm{v} / \mathrm{v})$ solution. Required amounts of lipid solutions were mixed in a $5 \mathrm{~mL}$ RBF and evaporated to dryness using a Laborota 400 rotary evaporator from Heidolph Instruments (Schwabach, Germany) creating a thin lipid layer on the wall of the flask (under vacuum, $280 \mathrm{rpm} / \mathrm{min}$ ). The films were hydrated with $20 \mathrm{mM}$ HBS buffer to obtain a lipid concentration of $6 \mathrm{mg} / \mathrm{mL}$, and the flasks were placed in a bath sonicator and allowed to equilibrate for 5 minutes. Then, the flasks were sonicated for 2 minutes until the lipid film was fully reconstituted. Liposomal suspension was further extruded through $200 \mathrm{~nm}$ and $100 \mathrm{~nm}$ polycarbonate membranes (Whatman) using an Avanti Mini Extruder (Avanti Polar Lipids) to obtain small unilamellar liposomes. For cellculture experiments, liposomes were filter-sterilised through a $0.22 \mu \mathrm{m}$ syringe filter and stored at $4^{\circ} \mathrm{C}$ up to two weeks.

2.6. Preparation of Lipoplexes. Liposomes and pDNA were diluted with OPTI-MEM. Equal volumes of pDNA solution and liposomal suspension were mixed and triturated several times to ensure homogeneous mixing. N/P ratio was calculated based on nitrogen containing DOTAP lipid $(699 \mathrm{~g} / \mathrm{mol})$ and the phosphorus containing phosphate group for $\mathrm{pDNA}$ $(330 \mathrm{~g} / \mathrm{mol})$. The mixture was allowed to stand for 20 minutes at room temperature (procedures were carried out under sterile conditions when preparing complexes for transfection studies).

2.7. Size and Zeta-Potential Measurements. Hydrodynamic diameter of liposomes and lipoplexes was determined by using Dynamic Light Scattering on a Malvern Zetasizer Nano ZS using Noninvasive Back Scatter (NIBS $\left.{ }^{\circledR}\right)$. Liposomes were diluted $(1: 10)$ with Milli-Q water and equilibrated to $25^{\circ} \mathrm{C}$ prior to measurement. Measurement subruns were set automatically by the device depending upon the sample. Liposomes and DNA were complexed at N/P of 2.5 for $20 \mathrm{~min}$ before measurement. Zeta potential of liposomes and lipoplexes was determined at conductivity $<100 \mu \mathrm{S} / \mathrm{cm}$. The zeta potential was calculated using the Smoluchowski equation.

2.8. Atomic Force Microscopy (AFM). Liposomes and lipoplexes were diluted $(1: 100)$ with Milli-Q water and pipetted onto a silicon wafer $\left(1 \times 1 \mathrm{~cm}^{2}\right)$. After $10 \mathrm{~min}$ of incubation, suspension was removed by aspirating the excess of water leaving liposomes and lipoplexes on the silica wafer. Atomic force microscopy was performed on a NanoWizard ${ }^{\circledR} 3$ NanoScience AFM from JPK Instruments (Berlin, Germany). The microscope was vibration-damped. Commercial 1-lever tips (NSC $14 \mathrm{Al} / \mathrm{BS}$ ) on a cantilever with a length of $125 \mu \mathrm{m}$ and a resonance frequency of about $160 \mathrm{~Hz}$ and force constant of $5 \mathrm{~N} / \mathrm{m}$ were used. All measurements were performed in tapping mode in air. Scan speed was adjusted between 0.5 and $1.5 \mathrm{~Hz}$.

2.9. Cryo-Scanning Electron Microscopy (Cryo-SEM). $10 \mu \mathrm{L}$ of liposomal sample was placed onto a sample holder and immersed in liquid nitrogen. The sample was fractured with a fine blade in the preparation chamber of a JEOL JSM-7500F (Jeol Ltd., Tokyo, Japan) and sputter-coated with platinum to increase conductivity. The sample was then transferred into the SEM chamber. The instrument was maintained at $-170^{\circ} \mathrm{C}$ all the time. Images were acquired at a voltage of $2.00 \mathrm{kV}$.

2.10. pDNA Intercalation Assay. $150 \mu \mathrm{L}$ of lipoplexes at increasing $\mathrm{N} / \mathrm{P}$ ratios was pipetted into an opaque 96microtiter plate. Subsequently, $50 \mu \mathrm{L}$ of gel-red dye was pipetted into the complex suspension and fluorescence was immediately measured at the appropriate wavelength.

\subsection{Toxicity Studies}

MTT. Toxicity studies, for comparison of several lipoplexes and $25 \mathrm{kDa}$ bPEI, were performed using the MTT (3-[4,5dimethylthiazol-2-yl]-2,5-diphenyltetrazolium bromide) reagent (Sigma-Aldrich, Taufkirchen, Germany). 10.000 cells/well were seeded in 96-well plates and incubated overnight before addition of samples. After $24 \mathrm{~h}$, the transfection reagents were aspirated and $200 \mu \mathrm{L}$ of $2 \mathrm{mg} / \mathrm{mL}$ MTT was added to each well, followed by further incubation of $3 \mathrm{~h}$. Then, $200 \mu \mathrm{L}$ of DMSO was added to dissolve the formazan crystals. The formazan solution was analysed at $580 \mathrm{~nm}$ using a spectrometer. Triton X-100, PBS buffer ( $\mathrm{pH}$ 7.4), and untreated cells were used as controls. Samples were analysed in triplicate.

$\mathrm{LDH}$. LDH assay was performed using LDH Cytotoxicity Kit (Roche Diagnostics, Basel, Switzerland). Cells were seeded at a density of 10.000 cells/well and incubated overnight. On the following day, cells were treated with samples for $24 \mathrm{~h}$. The supernatant was then transferred to a transparent 96well plate and the $\mathrm{LDH}$ assay was performed according to 
TABLE 1: Composition and physicochemical properties of polymer and liposomal formulations.

\begin{tabular}{lccc}
\hline Formulation $(\mathrm{mol}: \mathrm{mol})$ & Particle size \pm SD $[\mathrm{nm}]$ & AFM diameter $[\mathrm{nm}]$ & Zeta potential $[\mathrm{mV}]$ \\
\hline 25kDa bPEI & - & - & - \\
DOTAP & $84.7 \pm 3.4$ & 65.3 & +38.5 \\
hGDNT/DOTAP $(5: 95)$ & $89.8 \pm 6.9$ & 70.8 & +40.9 \\
PLFE/DOTAP $(5: 95)$ & $94.3 \pm 6.9$ & 63.6 & +38.1 \\
hGDNT/CH/DOTAP $(5: 45: 50)$ & $96.6 \pm 8.8$ & 71.3 & +41.1 \\
PLFE/CH/DOTAP $(5: 45: 50)$ & $103.7 \pm 49.4$ & 67.9 & +45.5 \\
hGDNT/PC/CH/DOTAP $(5: 30: 35: 30)$ & $96.6 \pm 8.8$ & +48.6 \\
\hline
\end{tabular}

the manufacturer's protocol. LDH activity was determined by monitoring the oxidation of pyruvate coupled with the reduction of NAD at $340 \mathrm{~nm}$.

2.12. Transfection Studies. SK-OV-3 cells were seeded at a density at 10.000 cells/well $24 \mathrm{~h}$ prior to transfection. Lipoplexes were added to cells at different $\mathrm{N} / \mathrm{P}$ ratios resulting in a pDNA amount of $0.5 \mu \mathrm{g}$ per well. Cells were incubated with lipoplexes for $4 \mathrm{~h}$ in IMDM medium to allow cellular uptake. The medium was then replaced by a fresh medium and the cells were further incubated for $48 \mathrm{~h}$. Cells were subsequently washed with $200 \mu \mathrm{L}$ PBS buffer and $50 \mu \mathrm{L}$ of lysis buffer was added and placed in an orbital shaker for $30 \mathrm{~min}$. Finally, $20 \mu \mathrm{L}$ of lysis buffer was transferred into an opaque 96-well microtiter plate. Expression of luciferase was measured by adding $50 \mu \mathrm{L}$ of luciferin to each well. Luminescence was expressed in RLU/mg (relative luminescence units/mg protein). The protein content was determined by pipetting $20 \mu \mathrm{L}$ of lysis buffer into a transparent 96-well plate. $200 \mu \mathrm{L}$ of bicinchoninic reagent was added and incubated for $30 \mathrm{~min}$ in an orbital shaker. In order to determine the protein content, the absorbance was measured at $562 \mathrm{~nm}$ and referenced against albumin standards.

2.13. Statistical Analysis. Data were expressed as mean \pm SD from four individual samples. Statistical analysis was performed by using two-tailed Student's $t$-test in Microsoft Excel. Significant differences were represented by $p<0.05$ $(*)$ and $p<0.01(* *)$.

\section{Results}

\subsection{Isolation, Purification, and Analysis of Tetraether Lipids}

3.1.1. Polar Lipid Fraction E (PLFE). The high polarity of the first solvent $\mathrm{MeOH}: \mathrm{H}_{2} \mathrm{O}(50: 50 \mathrm{v} / \mathrm{v})$ eluted mainly pigments, which fluoresced when exposed to UV light. The decreased polarity of the second eluent resulted in the PLFE. The final eluent $\mathrm{CHCl}_{3}: \mathrm{MeOH}(90: 10 \mathrm{v} / \mathrm{v})$ gave less polar compounds. The fraction containing PLFE was analysed by HPTLC, where silica gel was the stationary phase and the eluent was composed of $\mathrm{CHCl}_{3}: \mathrm{MeOH}: \mathrm{H}_{2} \mathrm{O}(70: 26: 4 \mathrm{v} / \mathrm{v})$. A spot at $R_{f} 0.2$ indicated the lipids (Figure 3 ).

3.1.2. Hydrolysed Lipid (hGDNT). The first solvent in the silica purification step $\mathrm{CHCl}_{3}$ eluted nonpolar substances like pigments (orange band). The second fraction which was eluted with $\mathrm{CHCl}_{3}: \mathrm{DE}(80: 20 \mathrm{v} / \mathrm{v})$ gave the less unipolar substance hGDNT where no nonitol group is present. hGDNT was finally obtained in the last step using $\mathrm{CHCl}_{3}: \mathrm{MeOH}(90: 10 \mathrm{v} / \mathrm{v})$ resulting in a dark brown band. hGDNT could be further purified by using cold acetone precipitation resulting in a resin-like appearance. The hydrolysed lipid hGDNT was analysed by HPTLC, where silica gel acted as a stationary phase and an eluent composed of $\mathrm{CHCl}_{3}: \mathrm{MeOH}(90: 10 \mathrm{v} / \mathrm{v})$ was used. One single spot indicated hGDNT at $R_{f} 0.2$ (Figure 4 ).

Mass spectrometry (ESI-MS) of hGDNT resulted in a sharp signal at $m / z 1479$, which indicated the $[\mathrm{M}+\mathrm{Na}]^{+}$ ion. Other characteristic signals are located at $m / z 751$ and 740 , which represent $[\mathrm{M}+2 \mathrm{Na}]^{2+}$ and $[\mathrm{M}+\mathrm{H}+\mathrm{Na}]^{2+}$ ions, respectively (Figure 5).

Recorded IR spectrum (Figure 5) indicates functional groups of hGDNT. A broad band at $3376 \mathrm{~cm}^{-1}$ corresponds to $\mathrm{OH}$ groups. Two sharp bands at $2921 \mathrm{~cm}^{-1}$ and $2854 \mathrm{~cm}^{-1}$ show $\mathrm{CH}, \mathrm{CH}_{2}$, and $\mathrm{CH}_{3}$ groups. Bands at $1460 \mathrm{~cm}^{-1}$ and $1276 \mathrm{~cm}^{-1}$ are evident for $\mathrm{CH} 2$ and $\mathrm{CH} 3$, respectively. C-O$\mathrm{C}$ (ether) and C-O-H (hydroxyl) groups are characterised by bands at $1102 \mathrm{~cm}^{-1}$ and $1085 \mathrm{~cm}^{-1}$, respectively. A sharp band at $758 \mathrm{~cm}^{-1}$ reveals $\mathrm{C}-\mathrm{H}$ bend.

$1 \mathrm{H}-\mathrm{NMR}$ spectrum of hydrolysed GDNT is in accordance with that reported by Lo and Chang [18]: $\delta$ 0.7-0.9 $\left(-\mathrm{CH}_{3}\right)$, 0.95-1.4 (-CH, $\left.-\mathrm{CH}_{2}\right), 1.45-1.9$ (cyclopentyl - $\left.\mathrm{CH}\right), 3.4-4.05$ $\left(-\mathrm{O}-\mathrm{CH},-\mathrm{O}-\mathrm{CH}_{2}\right.$ ) (Figure 6).

\subsection{Size Determination. See Tables 1 and 2.}

3.3. Atomic Force Microscopy (AFM). Morphological characterisation of tetraether lipid containing liposomes using AFM showed mostly round shaped vesicles at sizes ranging between $50 \mathrm{~nm}$ and $100 \mathrm{~nm}$ which correlate with DLS measurements. Liposomes appeared in irregular shapes and spread onto the silicon surface resulting in a lipid monolayer (Figure 8). Lipid-pDNA complexes for all liposomal formulations showed different structures, resembling the often proposed “onion-like" structures (Figure 9). In contrast to liposomes, lipoplexes did not spread onto the silicon surface.

3.4. Cryo-Scanning Electron Microscopy (Cryo-SEM). For liposome with tetraether lipids, only complete vesicles and no fracture planes were visible in Cryo-SEM, indicating the tight packaging of tetraether lipids within the lipid bilayer. 
TABLE 2: Composition and physicochemical properties of polyplexes and lipoplexes.

\begin{tabular}{lccc}
\hline Formulation (mol : mol) [N/P ratio] & Particle size \pm SD [nm] & AFM diameter [nm] & Zeta potential [mV] \\
\hline 25 kDa bPEI [5.0] & $60.2 \pm 10.2$ & 79.5 & +31.2 \\
DOTAP [2.5] & $220.1 \pm 26.8$ & 257.1 & +38.9 \\
hGDNT/DOTAP $(5: 95)[2.5]$ & $322.1 \pm 49.9$ & 317.4 & +41.0 \\
PLFE/DOTAP $(5: 95)[2.5]$ & $214.5 \pm 10.2$ & 210.7 & +42.7 \\
hGDNT/CH/DOTAP $(5: 45: 50)[2.7]$ & $450.1 \pm 4.5$ & 473.5 & +50.1 \\
PLFE/CH/DOTAP $(5: 45: 50)[2.7]$ & $372.3 \pm 15.0$ & 441.3 & +51.6 \\
hGDNT/PC/CH/DOTAP $(5: 30: 35: 30)[2.7]$ & $231.8 \pm 35.0$ & 398.4 & -48.6 \\
\hline
\end{tabular}

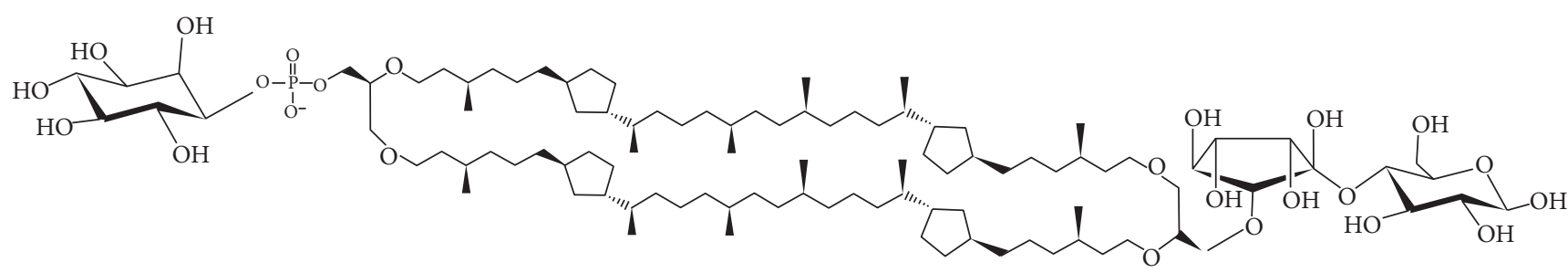

(a)

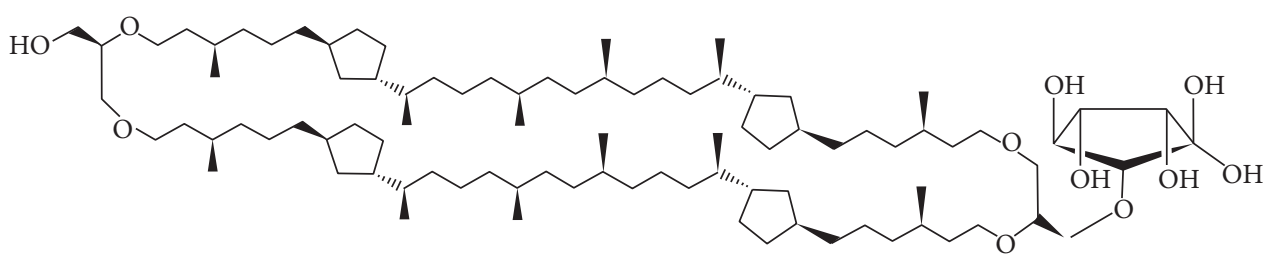

(b)

FIGURE 1: Structures of nonhydrolysed and hydrolysed fractions of tetraether lipids derived from Sulfolobus acidocaldarius. (a) PLFE and (b) hGDNT.

3.5. DNA Intercalation Assay. All liposomal formulations could complex pDNA in a concentration dependent manner until full complexation at N/P ratios of 2.5 to 3 . This correlates perfectly with the highest transfection efficiency in this range. DOTAP and $25 \mathrm{kDa}$ bPEI showed the most efficient complexation with pDNA, whereas all other liposomes were less effective in binding pDNA.

\subsection{Toxicity Studies. See Figures 12 and 13.}

3.7. Transfection Efficiency. Transfection studies with lipoplexes in SK-OV-3 cell line showed an overall reasonable transfection efficiency, which is comparable to the DOTAP standard. For binary mixtures, lipoplexes resulted in similar transfection efficiency to DOTAP. In contrast, incorporation of cholesterol and hGDNT into the bilayer resulted in an increase in transfection efficiency but decreased when PLFE was used as a stabilising agent. By incorporating PC and cholesterol, a 30\% decrease in transfection efficiency was observed.

\section{Discussion}

To our knowledge, this is the first study which investigates the potential of native highly purified tetraether lipids, derived from Sulfolobus acidocaldarius, for the transfection of mammalian cells. A standardised and reproducible extraction process for two different fractions of tetraether lipids from Sulfolobus acidocaldarius was established. By applying Soxhlet extraction of the freeze-dried biomass, the sugar moieties as well as the phosphate group of the tetraether lipids remained intact (Figure 1(a)). Followed by purification on a Chromabond C-18 column, PLFE could only be eluted with a mobile phase composed of $\mathrm{CHCl}_{3}: \mathrm{MeOH}: \mathrm{H}_{2} \mathrm{O}$ $(22.5: 55: 22.5 \mathrm{v} / \mathrm{v})$, demonstrating the high polarity of sugar groups in PLFE (Figure 1(a)). HPTLC analysis with a highly polar mobile phase revealed $R_{f}$ value of around 0.2 (Figure 3(b)), which is in accordance with Lo and Chang [18]. In contrast to the other findings, no further purification with methanol was necessary, because HPTLC analysis revealed a single spot for the PLFE fraction. Elferink et al. [19] had a range of spots in the corresponding fraction. Sugar moieties and the phosphate group could be removed by an acidic extraction process resulting in tetraether lipids with less polarity (Figures 1(b) and 4(a)). The procedure was more simplified in comparison to that of Bode et al. [20]. HPTLC analysis using a mobile phase of $\mathrm{CHCl}_{3}: \mathrm{MeOH}(90: 10 \mathrm{v} / \mathrm{v})$ revealed lower polarity in comparison to PLFE. $R_{f}$ value for hydrolysed lipids, which was around 0.3 , was compared to the hGDNT standard obtained from Surface \& Interface Technologies (Figure 4(b)). Further analysis of hydrolysed tetraether lipids by ESI-MS indicated a series of peaks around 


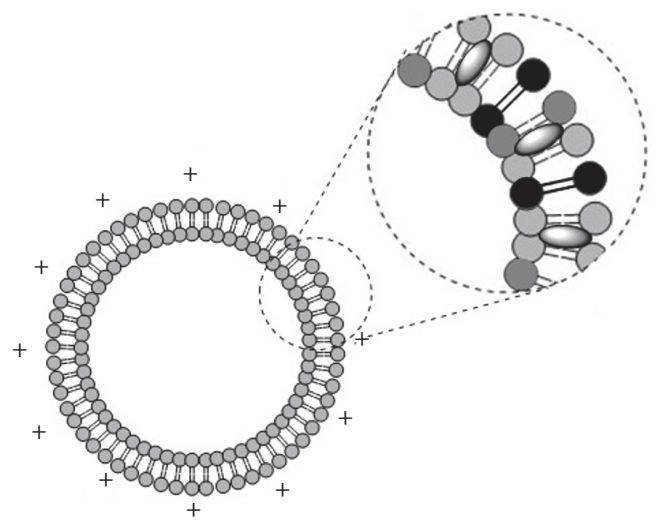

FIGURE 2: A model of a liposomal membrane containing tetraether lipids PLFE and hGDNT (black), complexing lipids DOTAP (dark grey colored), and helper lipids PC and CH (light grey, in between).

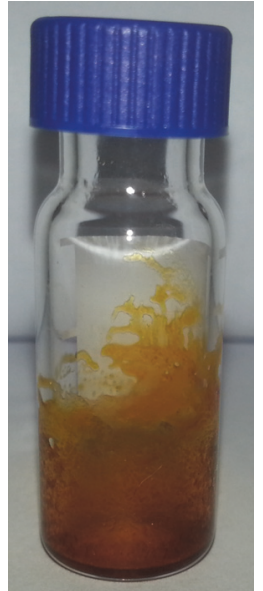

(a)

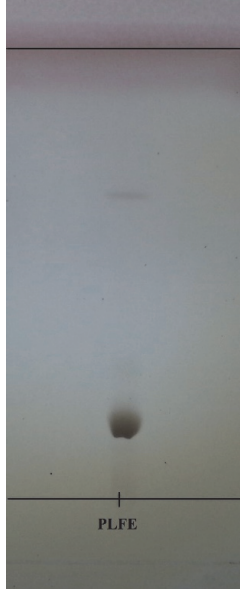

(b)

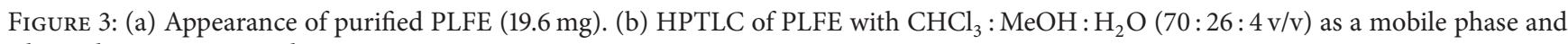
silica gel as a stationary phase.

the theoretical mass (Figure 5). This is due to the presence of cyclopentane rings in the molecule. For the hydrolysed lipid, the sodium ions could be detected. The molecular weight measured by mass spectrometry is $23 \mathrm{~g} / \mathrm{mol}$ above the theoretical masses, indicating $1.456 \mathrm{~g} / \mathrm{mol}$ for hGDNT. $1 \mathrm{H}-\mathrm{NMR}$ and IR spectrum of hGDNT (Figures 6 and 7) revealed relevant peaks and bands according to the analysis conducted by Parmentier et al. [21]. Preparation of liposomes, composed of tetraether lipids and DOTAP, was conducted at $5 \mathrm{~mol} \%$ to take advantage of the stabilising effect of tetraether lipids, which are incorporated into the lipid bilayer (Figure 2). A similar observation was also made by Jensen et al. [22] where the highest fraction of tetraether lipid was $18 \mathrm{~mol} \%$ in order to get stabilised liposomes. Furthermore, Parmentier et al. [23] stated that a molar fraction of 5-15 mol\% hGDNT was sufficient in delivering sensitive peptides through the GIT. The morphology of liposomes, which was analysed by Cryo-SEM, revealed round shaped vesicles with no visible fracture planes, which is common for conventional liposomes (Figure 10). The absence of fracture planes can be explained by the tight membrane packaging of liposomes with tetraether lipids as stabilising agents, which was investigated by Khan and Chong [24]. AFM images of lipoplexes indicate "onion-like" structures (Figure 9(b), inset), where a liposome builds a core and pDNA is enwrapped around it. Another layer of lipids is then attached on it, followed by another layer of pDNA [25]. The complexation efficacy determined by using gel-red assay revealed successful complexation of liposomes with pDNA at N/P ratios around $2-2.5$, where $80-90 \%$ of pDNA was complexed within the lipoplex (Figure 11). Superior complexation efficiency was observed in $25 \mathrm{kDa}$ bPEI due to the polycationic nature of the polymer favouring particles with sizes of $60 \mathrm{~nm}$ (Table 2 and Figure 9(a)). Transfection efficiency for $25 \mathrm{kDa}$ bPEI was high; however, the value is compared to liposomal DOTAP (Figure 14). This favours the usage of DOTAP over $25 \mathrm{kDa}$ bPEI due to the lower toxicity in the MTT assay, where only $5 \%$ of cells survived at the highest N/P ratio for the polymer and $40 \%$ for DOTAP (Figure 12) indicating high loss in the metabolic activity of the cell. Moreover, toxicity 


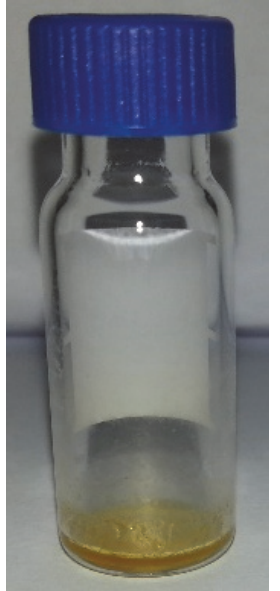

(a)

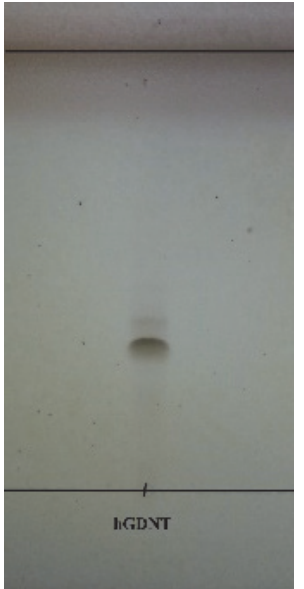

(b)

FIGURE 4: (a) Appearance of purified hGDNT (16.9 mg). (b) HPTLC of hGDNT with $\mathrm{CHCl}_{3}: \mathrm{MeOH}(90: 10 \mathrm{v} / \mathrm{v})$ as a mobile phase and silica gel as a stationary phase.

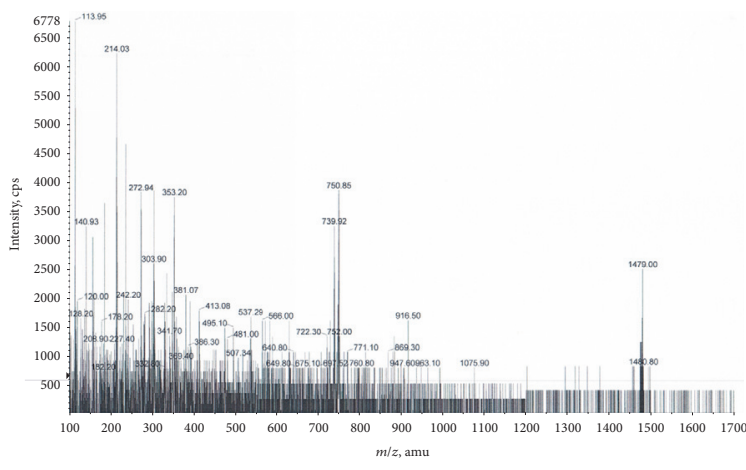

FIGURE 5: ESI-MS spectrum of purified hydrolysed lipid (hGDNT).

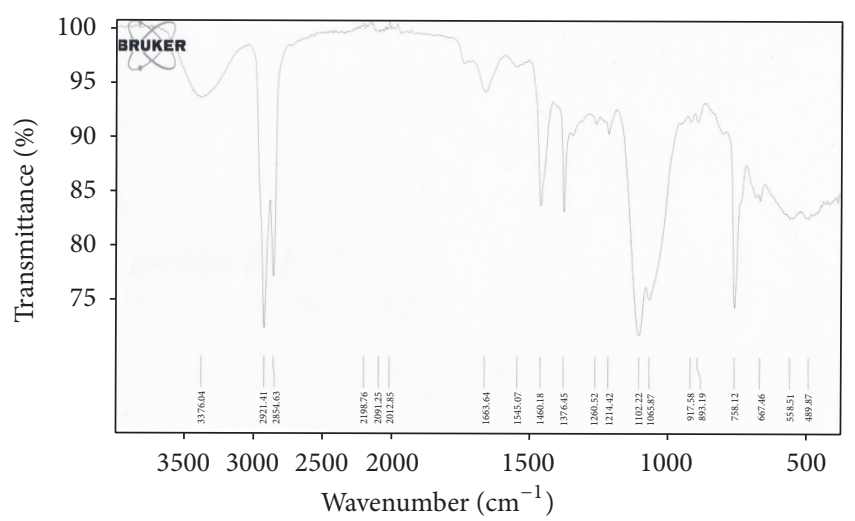

FIGURE 6: IR spectrum of hGDNT.

of $25 \mathrm{kDa}$ bPEI also affects the cell membrane resulting in higher leakage of lactate dehydrogenase (Figure 13) as seen in the LDH assay. The highest transfection efficiencies were obtained with lipoplexes with a positive zeta potential due to electrostatic effects that occur when lipoplexes interact with the negatively charged cell surface. A statistically significant

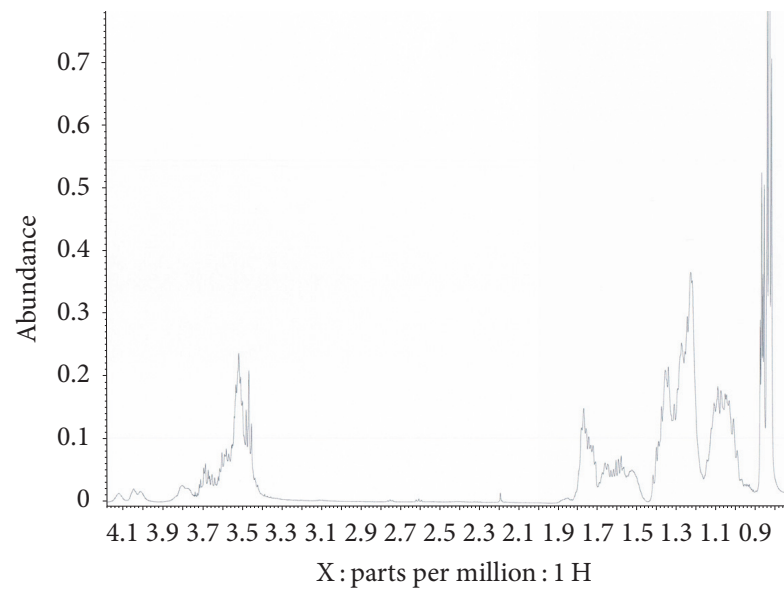

FIGURE 7: 1H-NMR spectrum of hGDNT.

transfection efficiency of PLFE/DOTAP (5:95) compared to DOTAP can be explained due to the stabilising effect of tetraether lipids, which prevents the denaturation of pDNA. A similar formulation hGDNT/DOTAP (5:95), where the sugar groups of the tetraether lipids have been removed, was less effective in delivering pDNA than PLFE/DOTAP (5:95). Considering almost equal diameters of both formulations of $214 \mathrm{~nm}$ and $322 \mathrm{~nm}$, respectively (Table 2), as well as similar zeta potentials, the stabilising of the hydrolysed tetraether lipid was too high, resulting in stronger retention of pDNA. By incorporating the helper lipid cholesterol and lowering the fraction of DOTAP, for example, PLFE/CH/DOTAP $(5: 45: 50)$ and hGDNT/CH/DOTAP $(5: 45: 50)$, transfection efficiency was significantly lower than the superior liposomal formulation. Both lipoplex formulations with cholesterol revealed larger diameters (Table 2) compared to the other formulations. Despite charge, the size of a lipoplex is crucial in obtaining high transfection efficiencies. Lipoplexes, 


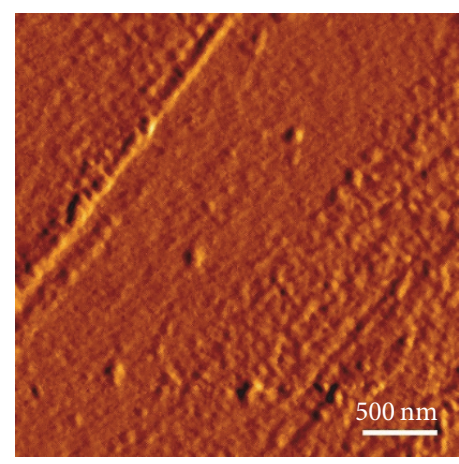

(a)

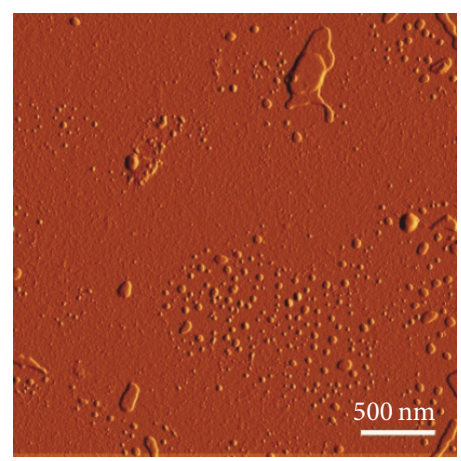

(d)

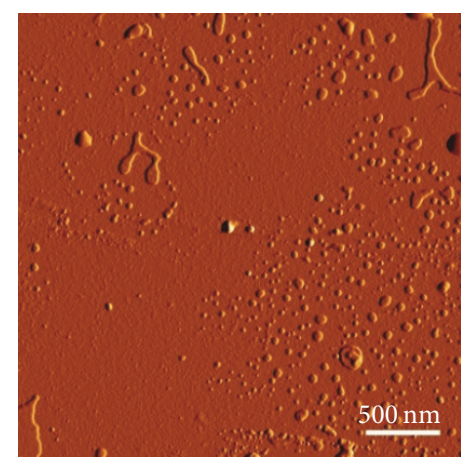

(b)

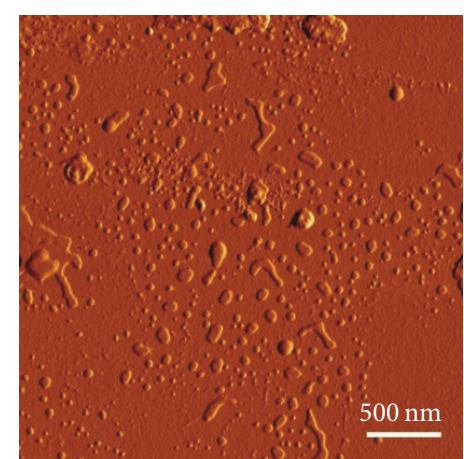

(e)

(g)

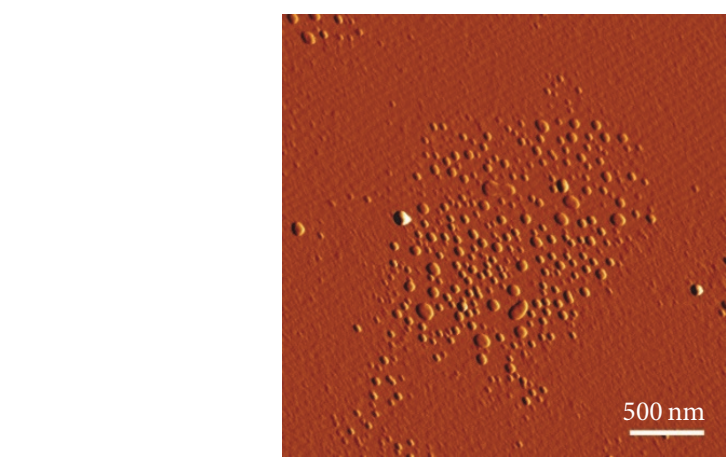

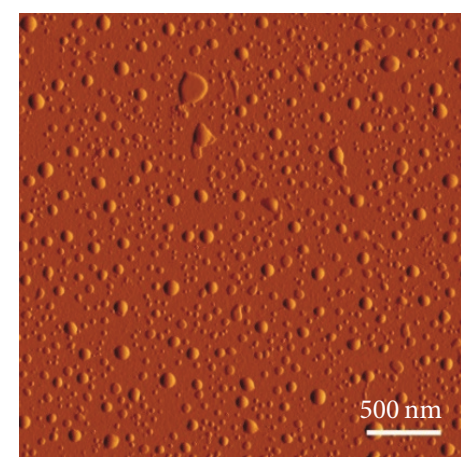

(c)

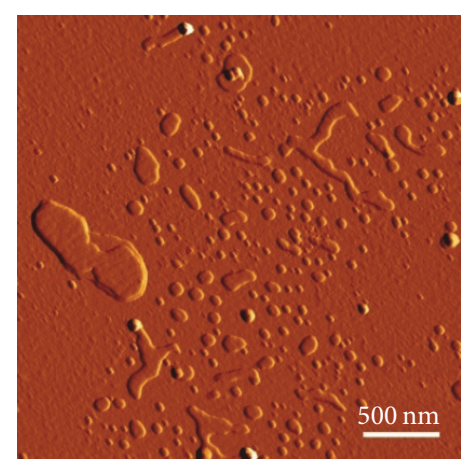

(f)

FIGURE 8: Visualisation of size and morphology of liposomes by atomic force microscopy. (a) $25 \mathrm{kDa}$ bPEI, (b) DOTAP, (c) hGDNT/DOTAP (5: 95), (d) PLFE/DOTAP ( $5: 95)$, (e) hGDNT/CH/DOTAP (5: $45: 50)$, (f) PLFE/CH/DOTAP ( $5: 45: 50)$, and (g) hGDNT/PC/CH/DOTAP $(5: 30: 35: 30)$.

in the size range of $214 \mathrm{~nm}$ to $322 \mathrm{~nm}$, showed the highest transfection efficiency due to the optimal surface, which promotes interaction with cell membrane. In the formulation comprising hGDNT/PC/CH/DOTAP (5:30:35:30), only $60 \%$ of pDNA could be complexed and protected (Figure 11). This confirms the high negative zeta potential of $-48.6 \mathrm{mV}$ (Table 1) indicating that the zeta potential is mainly determined by pDNA, which is enwrapped around the liposomes and in lipid tails with a diameter of around $10 \mathrm{~nm}$ (Figure $9(\mathrm{~g})$ ). Consequently, transfection efficiency was significantly lower compared to the other liposomal systems which was mainly due to the repulsive interaction of negatively charged lipoplexes with negative cell surface. This is in agreement with experiments from Tabatt et al. [26] who investigated novel lipid-DNA vectors, which had a negative surface charge. In creating a negatively charged lipoplex for gene delivery, thrombocyte or bile salt aggregation can be avoided when bringing the vehicle into the human body by injection or via the oral route [27]. Moreover, negatively charged particles can be used for the pulmonal delivery of genetic material without interacting with the mucous gel containing highly glycosylated segments.

\section{Conclusion}

Various tetraether lipids could be extracted, purified, and incorporated into spherical bilayers up to a certain amount resulting in liposomes that are nanoscaled and stable. 


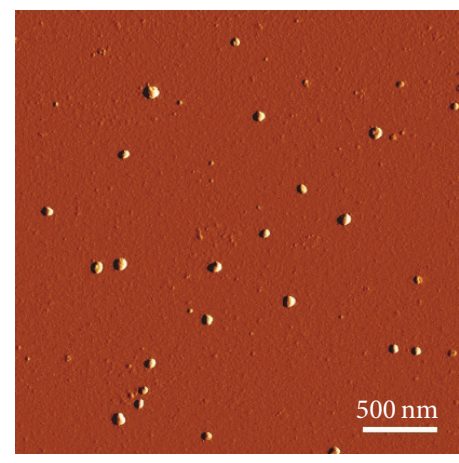

(a)

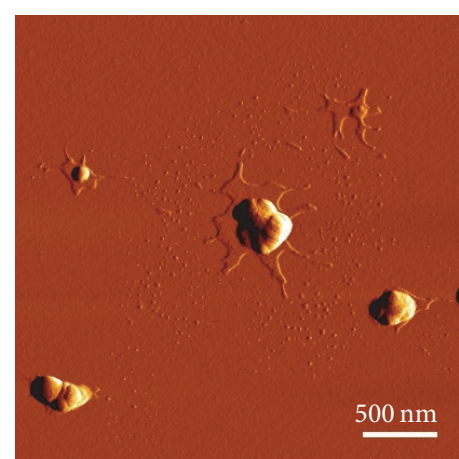

(d)

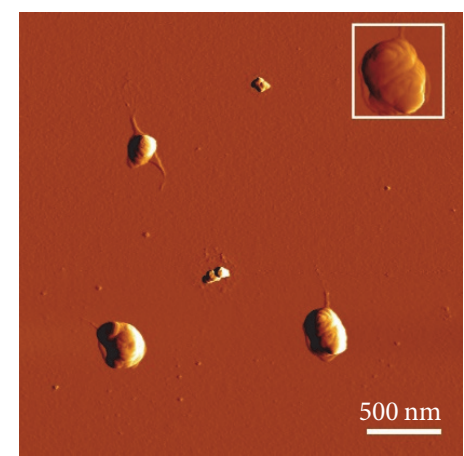

(b)

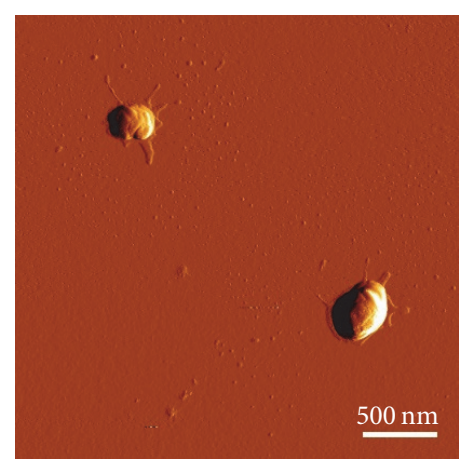

(e)

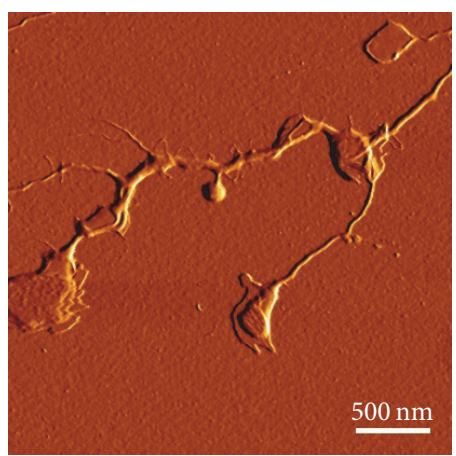

(g)

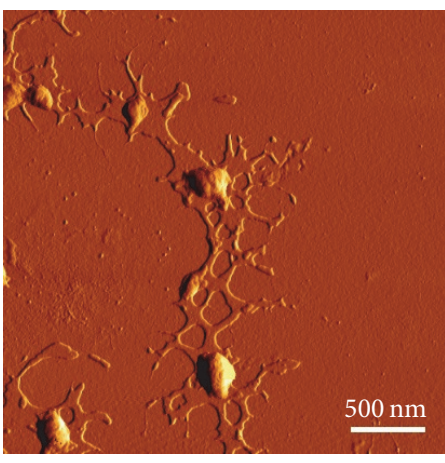

(c)

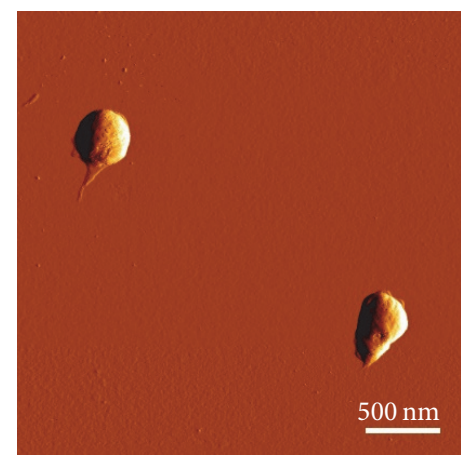

(f)

FIGURE 9: Visualisation of size and morphology of lipoplexes by atomic force microscopy. (a) $25 \mathrm{kDa}$ bPEI, (b) DOTAP, (c) hGDNT/DOTAP (5: 95), (d) PLFE/DOTAP ( $5: 95)$, (e) hGDNT/CH/DOTAP $(5: 45: 50)$, (f) PLFE/CH/DOTAP $(5: 45: 50)$, and (g) hGDNT/PC/CH/DOTAP $(5: 30: 35: 30)$. All formulations were complexed at N/P 2.5-2.7. Inset: $500 \times 500 \mathrm{~nm}$.

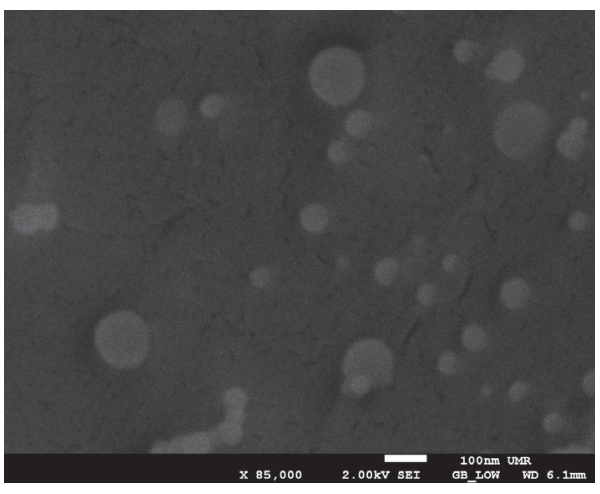

(a)

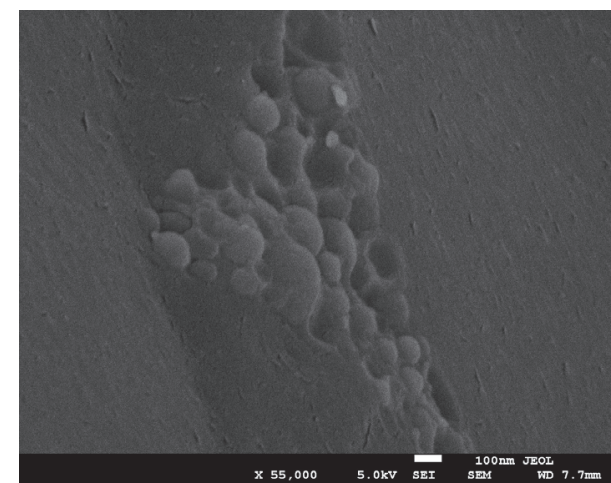

(b)

FIGURE 10: Cryo-SEM images of tetraether lipid containing liposomes. (a) hGDNT/DOTAP (5:95) and (b) hGDNT/PC/CH/DOTAP $(5: 30: 35: 30)$. 


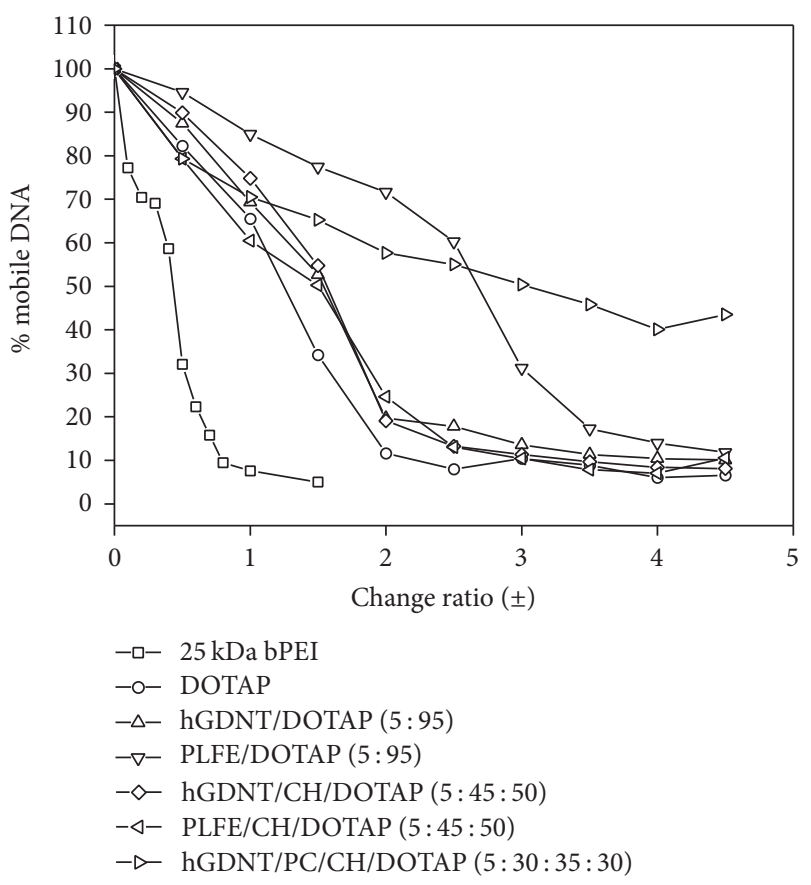

FIgURE 11: pDNA immobilisation of lipoplexes at increasing N/P $(+/-)$ ratios. DNA complexes with increasing amounts of tetraether lipids (PLFE or hGDNT) were prepared and analysed with gel-red (1x).

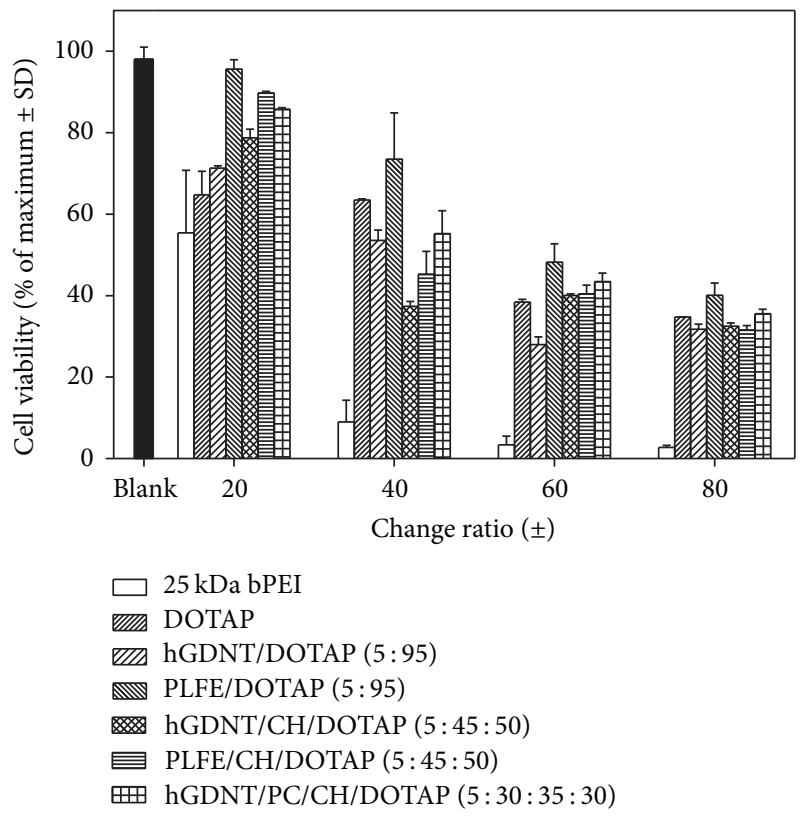

FIGURE 12: MTT assay of tetraether lipid containing lipoplexes and polyplexes.

Transfection agents based on tetraether lipids could be easily prepared by adding the cationic lipid DOTAP and pDNA resulting in stable and positively charged lipoplexes. In this study, we have shown that lipoplexes containing tetraether lipids have a statistically significant higher transfection efficiency compared to DOTAP alone. Moreover, a formulation

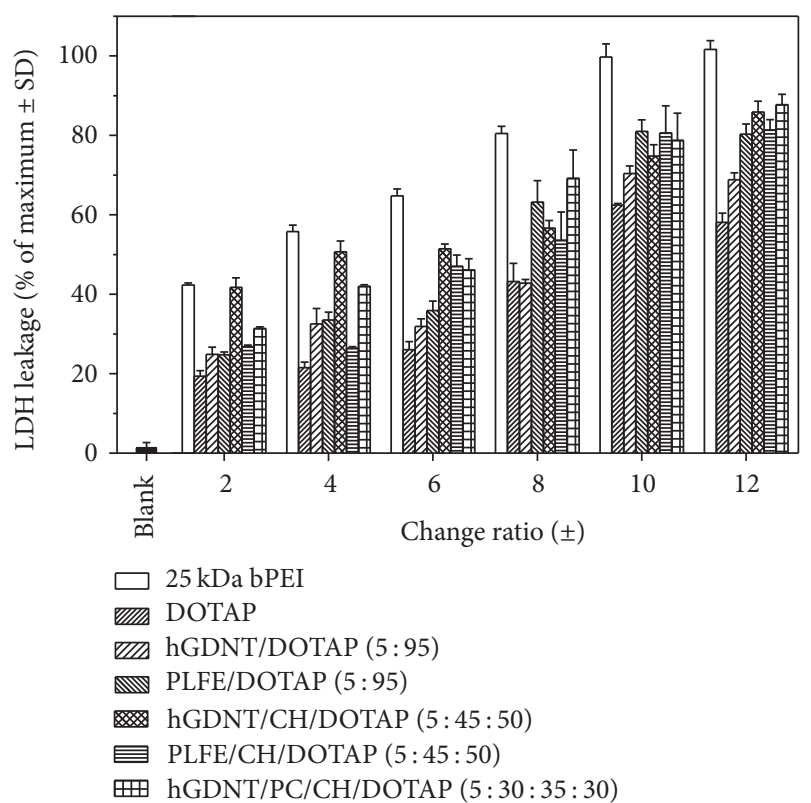

FIGURE 13: LDH assay of tetraether lipid containing lipoplexes and polyplexes.

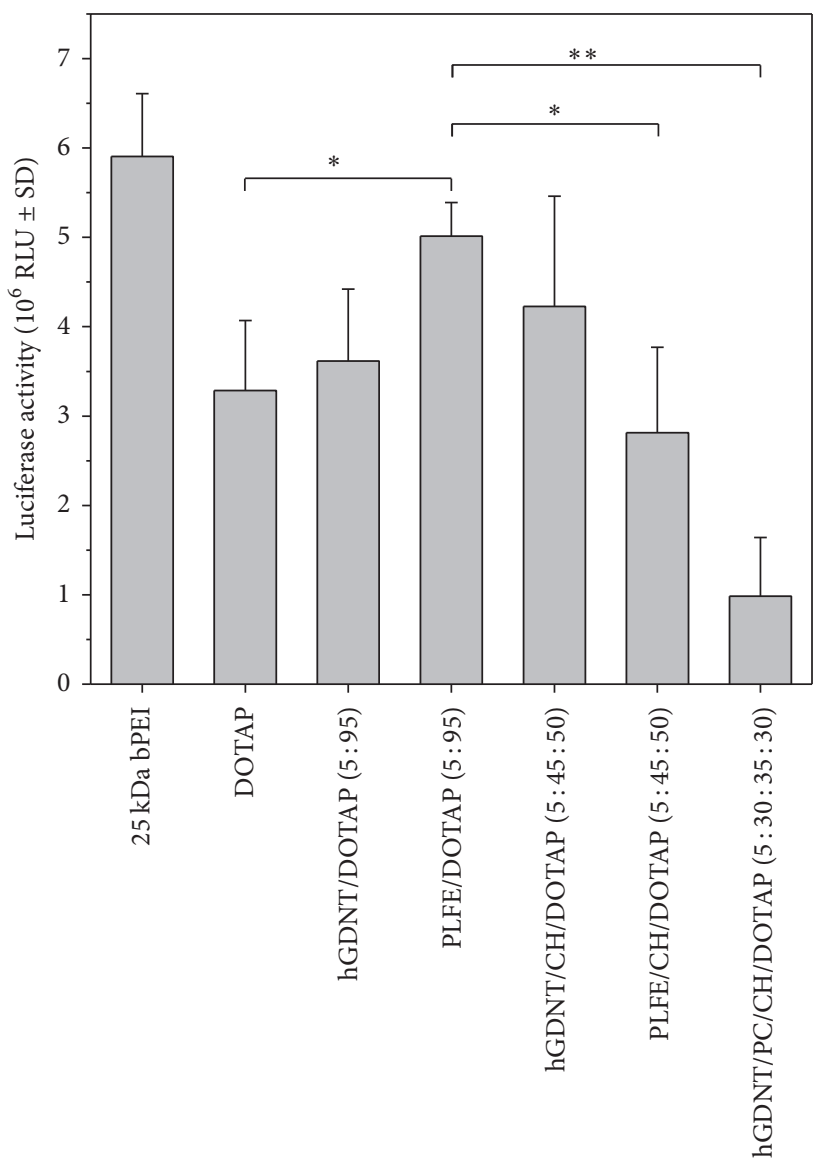

FIGURE 14: Transfection efficiency of various formulations. ${ }^{*} p<$ $0.05 ;{ }^{* *} p<0.01$. 
was identified that has the potential to be used for the oral gene therapy by circumventing platelet and bile salt aggregation.

\section{Competing Interests}

The authors declare that there are no competing interests regarding the publication of this paper.

\section{Acknowledgments}

The authors gratefully thank the SiT (Surface \& Interface Technologies, Rosenhof GmbH, Heiligenstadt, Germany) for kindly providing the freeze-dried biomass of Sulfolobus acidocaldarius.

\section{References}

[1] J. H. Felgner, R. Kumar, C. N. Sridhar et al., "Enhanced gene delivery and mechanism studies with a novel series of cationic lipid formulations," The Journal of Biological Chemistry, vol. 269, no. 4, pp. 2550-2561, 1994.

[2] C. L. Kneuer, C. Ehrhardt, U. Bakowsky et al., "The influence of physicochemical parameters on the efficacy of non-viral DNA transfection complexes: a comparative study," Journal of Nanoscience and Nanotechnology, vol. 6, no. 9-10, pp. 27762782, 2006.

[3] N. Nayerossadat, P. Ali, and T. Maedeh, "Viral and nonviral delivery systems for gene delivery," Advanced Biomedical Research, vol. 1, article 27, 2012.

[4] A. Sharma and U. S. Sharma, "Liposomes in drug delivery: progress and limitations," International Journal of Pharmaceutics, vol. 154, no. 2, pp. 123-140, 1997.

[5] G. Caetano-Anollés, A. Nasir, K. Zhou et al., "Archaea: the first domain of diversified life," Archaea, vol. 2014, Article ID 590214, 26 pages, 2014.

[6] A. Spang, J. Martijn, J. H. Saw, A. E. Lind, L. Guy, and T. J. G. Ettema, "Close encounters of the third domain: the emerging genomic view of archaeal diversity and evolution," Archaea, vol. 2013, Article ID 202358, 12 pages, 2013.

[7] B. Chaban, S. Y. M. Ng, and K. F. Jarrell, "Archaeal habitatsfrom the extreme to the ordinary," Canadian Journal of Microbiology, vol. 52, no. 2, pp. 73-116, 2006.

[8] A. Jacquemet, J. Barbeau, L. Lemiègre, and T. Benvegnu, "Archaeal tetraether bipolar lipids: structures, functions and applications," Biochimie, vol. 91, no. 6, pp. 711-717, 2009.

[9] P. L.-G. Chong, U. Ayesa, V. P. Daswani, and E. C. Hur, "On physical properties of tetraether lipid membranes: effects of cyclopentane rings," Archaea, vol. 2012, Article ID 138439, 11 pages, 2012.

[10] M. J. Hanford and T. L. Peeples, "Archaeal tetraether lipids: unique structures and applications," Applied Biochemistry and Biotechnology-Part A Enzyme Engineering and Biotechnology, vol. 97, no. 1, pp. 45-62, 2002.

[11] Z. Li, J. Chen, W. Sun, and Y. Xu, "Investigation of archaeosomes as carriers for oral delivery of peptides," Biochemical and Biophysical Research Communications, vol. 394, no. 2, pp. 412417, 2010.

[12] E. Moghimipour, M. Kargar, Z. Ramezani, and S. Handali, “The potent in vitro skin permeation of archaeosome made from lipids extracted of Sulfolobus acidocaldarius," Archaea, vol. 2013, Article ID 782012, 7 pages, 2013.

[13] G. Mahmoud, J. Jedelská, B. Strehlow, and U. Bakowsky, "Bipolar tetraether lipids derived from thermoacidophilic archaeon Sulfolobus acidocaldarius for membrane stabilization of chlorin e6 based liposomes for photodynamic therapy," European Journal of Pharmaceutics and Biopharmaceutics, vol. 95, pp. 88-98, 2015.

[14] A. Ozcetin, E. Dayyoub, C. Hobler, M. Keusgen, and U. Bakowsky, "Selective interactions of concanavalin A-modified tetraether lipid liposomes," Physica Status Solidi (C), vol. 8, no. 6, pp. 1985-1989, 2011.

[15] M. Brard, C. Lainé, G. Réthoré et al., "Synthesis of archaeal bipolar lipid analogues: a way to versatile drug/gene delivery systems," Journal of Organic Chemistry, vol. 72, no. 22, pp. 82678279, 2007.

[16] G. Réthoré, T. Montier, T. Le Gall et al., "Archaeosomes based on synthetic tetraether-like lipids as novel versatile gene delivery systems," Chemical Communications, no. 20, pp. 2054-2056, 2007.

[17] A. Attar, A. Ogan, S. Yucel, and K. Turan, "The potential of archaeosomes as carriers of pDNA into mammalian cells," Artificial Cells, Nanomedicine and Biotechnology, vol. 44, no. 2, pp. 710-716, 2016.

[18] S.-L. Lo and E. L. Chang, "Purification and characterization of a liposomal-forming tetraether lipid fraction," Biochemical and Biophysical Research Communications, vol. 167, no. 1, pp. 238243, 1990.

[19] M. G. L. Elferink, J. G. de Wit, R. Demel, A. J. M. Driessen, and W. N. Konings, "Functional reconstitution of membrane proteins in monolayer liposomes from bipolar lipids of Sulfolobus acidocaldarius," The Journal of Biological Chemistry, vol. 267, no. 2, pp. 1375-1381, 1992.

[20] M. L. Bode, S. R. Buddoo, S. H. Minnaar, and C. A. du Plessis, "Extraction, isolation and NMR data of the tetraether lipid calditoglycerocaldarchaeol (GDNT) from Sulfolobus metallicus harvested from a bioleaching reactor," Chemistry and Physics of Lipids, vol. 154, no. 2, pp. 94-104, 2008.

[21] J. Parmentier, B. Thewes, F. Gropp, and G. Fricker, "Oral peptide delivery by tetraether lipid liposomes," International Journal of Pharmaceutics, vol. 415, no. 1-2, pp. 150-157, 2011.

[22] S. M. Jensen, C. J. Christensen, J. M. Petersen, A. H. Treusch, and M. Brandl, "Liposomes containing lipids from Sulfolobus islandicus withstand intestinal bile salts: an approach for oral drug delivery?" International Journal of Pharmaceutics, vol. 493, no. 1-2, pp. 63-69, 2015.

[23] J. Parmentier, M. M. M. Becker, U. Heintz, and G. Fricker, "Stability of liposomes containing bio-enhancers and tetraether lipids in simulated gastro-intestinal fluids," International Journal of Pharmaceutics, vol. 405, no. 1-2, pp. 210-217, 2011.

[24] T. K. Khan and P. L.-G. Chong, "Studies of archaebacterial bipolar tetraether liposomes by perylene fluorescence," Biophysical Journal, vol. 78, no. 3, pp. 1390-1399, 2000.

[25] J. Sitterberg, A. Özcetin, C. Ehrhardt, and U. Bakowsky, "Utilising atomic force microscopy for the characterisation of nanoscale drug delivery systems," European Journal of Pharmaceutics and Biopharmaceutics, vol. 74, no. 1, pp. 2-13, 2010.

[26] K. Tabatt, C. Kneuer, M. Sameti et al., "Transfection with different colloidal systems: comparison of solid lipid nanoparticles and liposomes," Journal of Controlled Release, vol. 97, no. 2, pp. 321-332, 2004. 
[27] A. N. Ilinskaya and M. A. Dobrovolskaia, "Nanoparticles and the blood coagulation system. Part II: safety concerns," Nanomedicine, vol. 8, no. 6, pp. 969-981, 2013. 

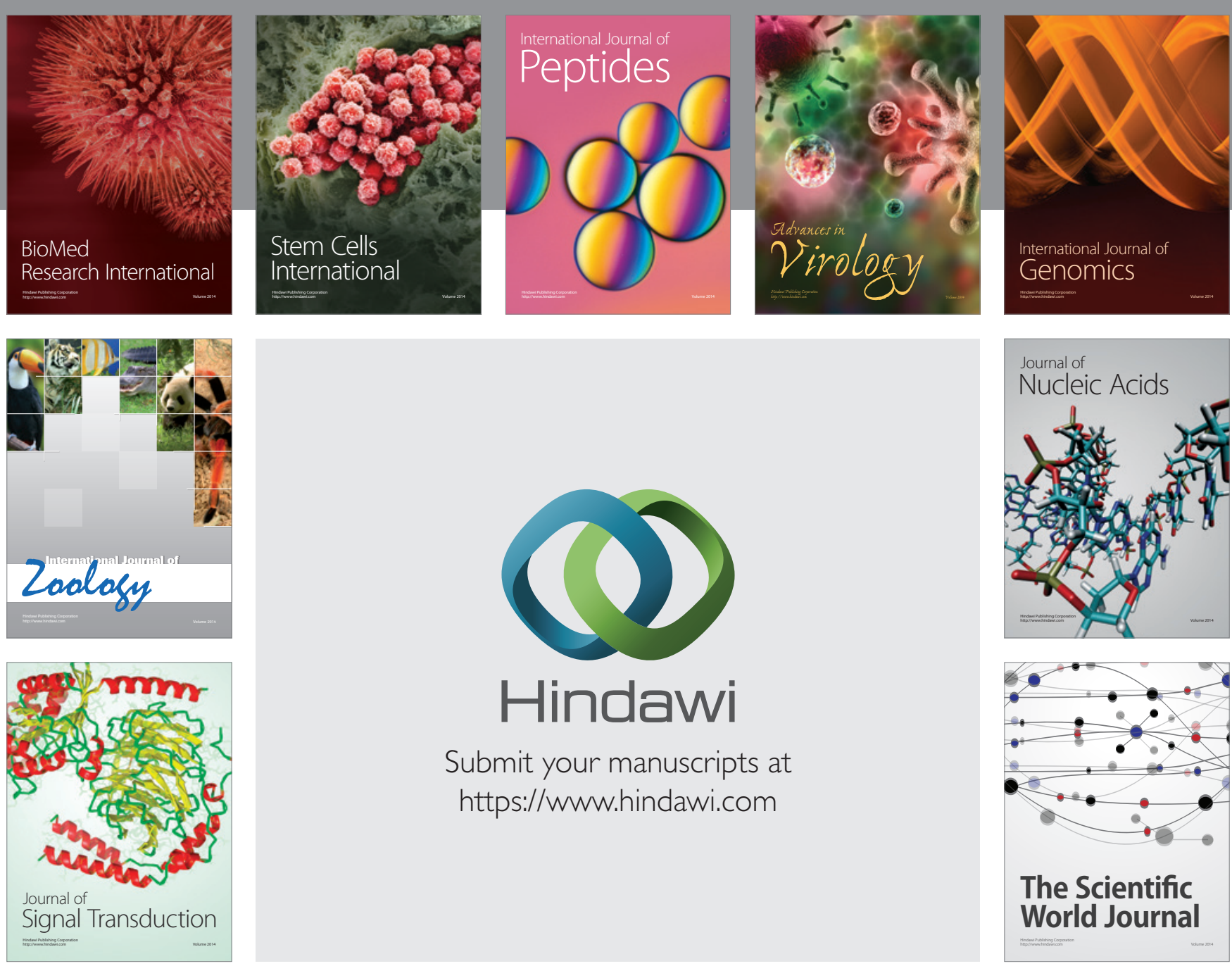

Submit your manuscripts at

https://www.hindawi.com
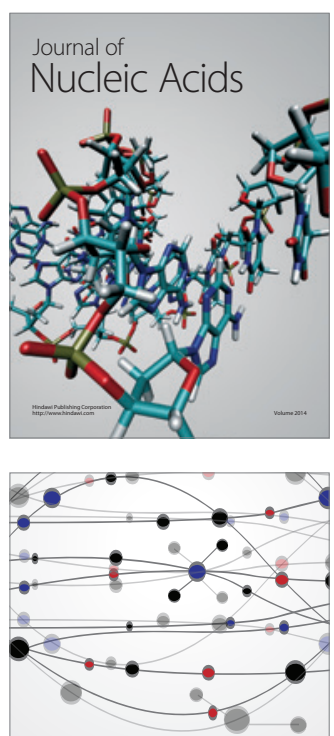

The Scientific World Journal
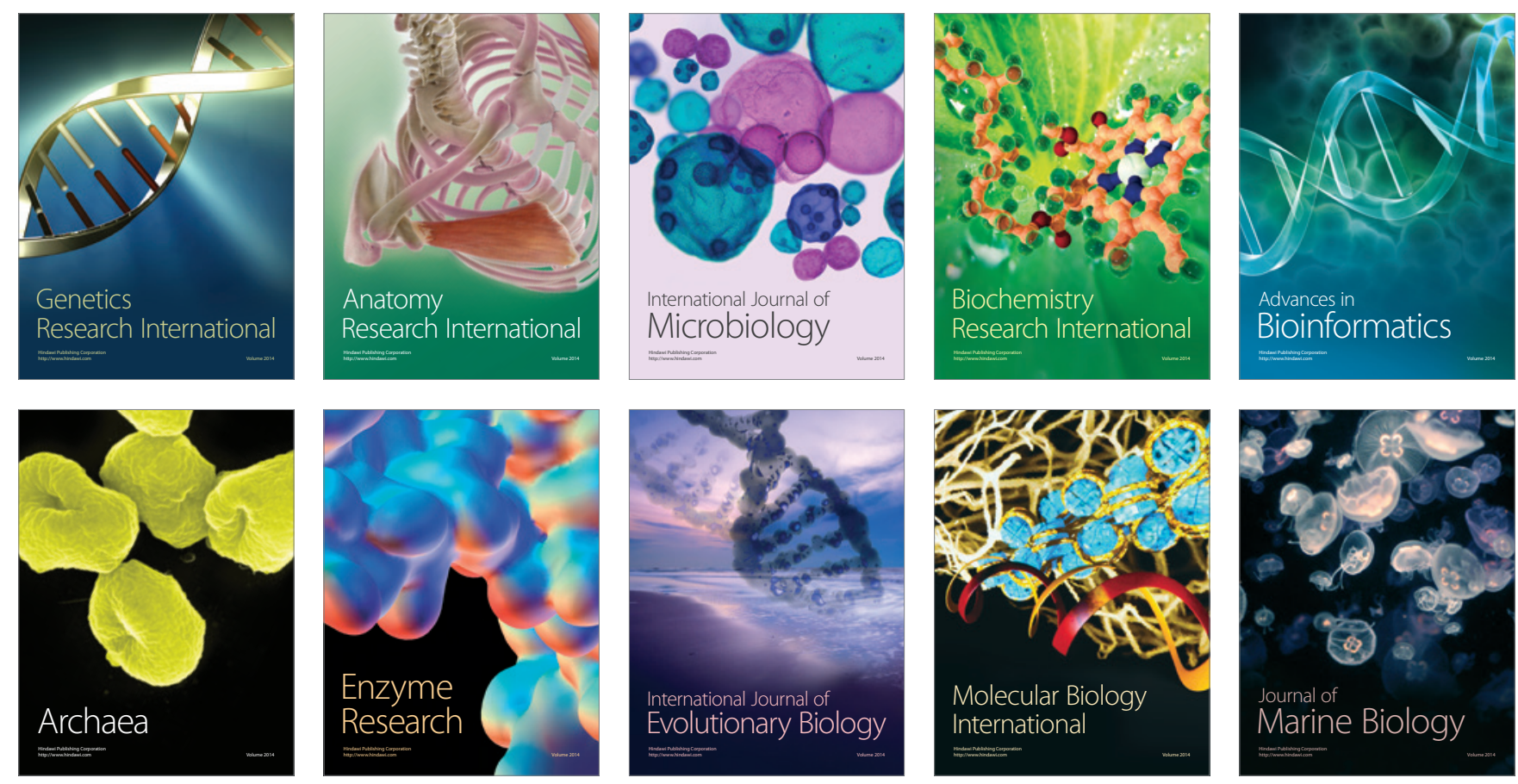